\title{
The Potential Benefits of Renal Replacement Therapy in Combination with a Hemadsorption Column in Critically III Patients with Acute on Chronic Liver Failure
}

\section{Tomescu Dana}

Institutul Clinic Fundeni

Mihai Popescu ( $\square$ lcf4ever@yahoo.com)

Institutul Clinic Fundeni https://orcid.org/0000-0002-2947-335X

Gheorghe Liliana

Institutul Clinic Fundeni

lacob Speranța

Institutul Clinic Fundeni

Dima Simona

Institutul Clinic Fundeni

Popescu Irinel

Institutul Clinic Fundeni

\section{Research article}

Keywords: extracorporeal liver support, hemadsorption, liver failure, liver cirrhosis, liver transplantation, inflammation

Posted Date: September 15th, 2020

DOI: https://doi.org/10.21203/rs.3.rs-54770/v1

License: (c) (i) This work is licensed under a Creative Commons Attribution 4.0 International License. Read Full License 


\section{Abstract}

Background. Acute on chronic liver failure (AoCLF) represents a life-threatening complication of liver cirrhosis with high mortality if patients cannot be bridged to emergency liver transplantation. The aim of this study was to assess clinical and paraclinical effects of renal replacement therapy (RRT) in combination with a hemadsorption column in patients with AoCLF.

Methods. Patients were included in the study after Intensive Care Unit (ICU) admission and RRT in combination with CytoSorb ${ }^{\circledR}$ was started for three consecutive sessions. Clinical and paraclinical data were recorded before the first session and after the end of the third session. 28-days mortality was also noted.

Results. Fourteen patients were included in the final data analysis. The use of CytoSorb ${ }^{\circledR}$ was associated with a decrease in bilirubin levels $(p=0.03)$ and creatinine $(p=0.02)$ and an increase in urine output $(p=$ $0.02)$. Although we observed a significant decrease in platelet count $(p=0.05)$, no haemorrhagic complications were noted. C-Reactive Protein significantly decreased after the therapy $(p=0.05)$, but we did not observe a similar decrease in leucocyte count $(p=0.87)$ or procalcitonin levels $(p=0.18)$. Seven patients underwent emergency liver transplantation and survival was $100 \%$ in this group.

Conclusion. The use of CytoSorb ${ }^{\circledR}$ was associated with an improvement in liver and renal functions and a decrease in $\mathrm{C}$ - Reactive Protein. Thrombocytopenia represents the main adverse effect of the therapy, but no haemorrhagic complications were recorded. In patients that could be bridged to liver transplantation, survival was $100 \%$.

Trial registration number: NCT04511507

\section{Background}

Acute on Chronic Liver Failure (AoCLF) represent life-threatening complication of cirrhosis associated with multiple system organ dysfunction and high mortality. To date, liver transplantation remains the only definitive treatment [1]. Nevertheless, due to the scarcity of liver grafts, such patients must be treated in a dedicated liver intensive care unit (ICU) and bridged until a suitable graft is found.

Recent studies have demonstrated that systemic inflammation represents the hallmark of AoCLF [2]. Patients demonstrate an inflammatory pattern of increased pro-inflammatory and decreased in antiinflammatory cytokines that is apparently correlated with the severity of organ dysfunction and mortality [3]. The theory that rebalancing inflammation in AoCLF patients has focused on the effects of different liver support therapies on clinical or laboratory parameters, as well as on survival benefits, without reaching positive results. Unfortunately, the most frequent use liver support devices - the MARS system and the Prometheus system - could not improve patient outcome and survival in AoCLF patients $[4,5]$. The reason for this failure to rescue may be that, even though cytokines are theoretically cleared with 
both systems, their production rate in AoCLF may out weight the possibility of removal by the two systems [6].

CytoSorb ${ }^{\circledR}$ is a hemadsorption column that has proved its efficacy in removing pro-inflammatory cytokines and re-balancing the inflammatory response syndrome in different clinical settings [7, 8]. Our hypothesis is that the use of RRT alongside hemoadsorption may modulate the severe inflammation associated with AoCLF and thus improve liver functional tests and clinical outcome.

\section{Methods}

The ethical approval and study registration were provided by the Ethical Committee of Fundeni Clinical Institute, Bucharest, Romania and the study was performed in accordance with the Declaration of Helsinki. The aim of this study was to assess clinical and paraclinical effects of a hemadsorption column (CytoSorb ${ }^{\circledR}$ ) on liver functional tests, severity scores and 28 days survival in patients with AoCLF admitted in the general ICU of a university hospital.

All patients aged above 18 years old diagnosed with AoCLF, defined in accordance with the study of Moreau et al. [9], admitted to the ICU over a 30 months period (between January 2017 and July 2019) who had at least two SIRS (Severe Inflammatory Response Syndrome) criteria [10] and one organ dysfunction were included in the present study. Organ dysfunctions were determined based on the criteria used in the Clif-SOFA score (Sequential Organ Failure Assessment). Exclusion criteria consisted of death within 24 hours after admission and contra-indication to RRT.

After study inclusion, high-flow ( $65 \mathrm{~mL} / \mathrm{kg}$ body weight) continuous venovenous hemodiafiltration (CVVHDF) in combination with hemoadsorption was applied to all patients. Standard anticoagulation with unfractionated heparin was used to prevent circuit clotting. A single session was considered a 24hour CVVHDF procedure. If the CVVHDF procedure lasted for less than 24 hours due to technical malfunction, another procedure was applied within the next two hours and the treatment continued for the remaining time. If the procedure could not be reinitiated within two hours the patient was excluded from the study. A complete treatment cycle consisted of three consecutive CVVHDF sessions. If a complete cycle could not be performed due to severe coagulopathy, the patient was excluded from the study.

The following data were collected after the patient was included in the study: age, sex, number of SIRS criteria, number of organ dysfunctions. Before the treatment was started, the following data were collected: heart rate, systolic and mean arterial blood pressure, $\mathrm{PaO}_{2} / \mathrm{FiO}_{2}$ ratio (arterial partial pressure of oxygen / fraction of inspired oxygen), serum creatinine, total bilirubin, leukocyte count, platelet count, haemoglobin, haematocrit, urine output, Glasgow coma scale, $\mathrm{C}$ reactive protein, procalcitonin and vasopressor support. At the end of the treatment the following data were collected: systolic and mean arterial pressure, $\mathrm{PaO}_{2} / \mathrm{FiO}_{2}$ ratio, serum creatinine, total bilirubin, leukocyte count, platelet count, haemoglobin, haematocrit, urine output, Glasgow coma scale, $\mathrm{C}$ reactive protein, procalcitonin and 
vasopressor support. Clif-SOFA score and Clif-C AoCLF score were calculated before the treatment and Clif-SOFA score was calculated after the three consecutive sessions. The follow-up was performed at 28 days and ICU length of stay and mortality were noted.

Statistical analysis. Statistical analyses were performed using SPSS 19.0 (SPSS Inc®, Chicago, IL, USA). Data are presented as mean \pm standard deviation of the mean, median (min, max) otherwise percentage. Data distribution was examined to insure the proper statistical examination. Categorical variables were analysed with Chi-square test and quantitative data were analysed with independent samples t-test. Mann-Whitney test was used when the analysed data did not follow a normal distribution. Survival was assessed using Kaplan-Meier analysis and the $\mathrm{P}$ value was derived using log-rank test. All $P$ values are two-tailed and a $\mathrm{P}$ value of less than 0.05 was considered statistically significant.

\section{Results}

Eighteen patients fulfilled the inclusion criteria and fourteen were included in the final analysis. Four patients were excluded from the study due to inability to reinitiate the CVVHDF session within two hours after circuit clotting. The mean age in the study group was $50.5 \pm 11.7$ years. The causes of liver failure were: liver cirrhosis due to hepatitis B virus -7 patients (50\%), liver cirrhosis due to hepatitis $C$ virus - 5 patients (35.8\%), one patient alcoholic liver cirrhosis (7.1) and one patient non-alcoholic steatohepatitis (7.1\%). The median number of SIRS criteria was $2[2,4]$ and the median number of organ dysfunctions was $3[2,5]$. The mean Clif-SOFA score at ICU admission was $12.6 \pm 1.9$ and the mean Clif-C ACLF score was $55.2 \pm 9.1$. According to their severity scores, five patients $(35.8 \%)$ had a grade 2 AoCLF and the rest $(\mathrm{n}=9)$ had grade 3 AoCLF.

Statistical analysis showed a significant decrease in creatinine levels $(p=0.02)$ and an increase in urine output $(p=0.02)$ and a decrease in bilirubin levels $(p=0.03)$ after the treatment. Although platelet count $(p=0.05)$ also decreased, there were no haemorrhagic complications associated with the therapy and we did not record a significant decrease in haemoglobin levels $(p=0.86)$ and haematocrit $(p=0.92)$. Three patients $(27.3 \%)$ required vasopressor support before the treatment and the same patients required vasopressor support after the treatment with a non-significant increase in noradrenaline dose $(p=0.86)$. A significant decrease in C-reactive Protein $(p=0.05)$ was recorded. There was a non-significant decrease in white blood cells count $(p=0.87)$ and a non-significant increase in procalcitonin levels $(p=0.18)$. Data are presented in Table 1. 
Table 1

Clinical and paraclinical comparison of data collected before and after RRT with CytoSorb® was applied; RRT - renal replacement therapy

\begin{tabular}{|c|c|c|c|}
\hline Parameter & Pre-RRT & Post-RRT & $P$ value \\
\hline Mean arterial pressure $(\mathrm{mmHg})$ & $72 \pm 13$ & $72 \pm 11$ & 0.64 \\
\hline $\mathrm{PaO} 2 / \mathrm{FiO} 2$ & $281 \pm 114$ & $308 \pm 117$ & 0.77 \\
\hline Creatinine $(\mathrm{mg} / \mathrm{dL})$ & $1.29 \pm 0.88$ & $0.92 \pm 0.35$ & $0.02^{*}$ \\
\hline Urine output (mg/dL) & $1085[100,3450]$ & $2010[140,8500]$ & $0.02^{\star}$ \\
\hline Total bilirubin (mg/dL) & $18.02 \pm 13.37$ & $12.15 \pm 9.27$ & $0.03^{*}$ \\
\hline Platelet count $\left(/ \mathrm{mm}^{3}\right)$ & $100000[33000,193000]$ & $46500[11000,128000]$ & $0.05^{\star}$ \\
\hline Glasgow coma scale (points) & $13.5[6,15]$ & $15[3,15]$ & 0.58 \\
\hline White blood cell count $(/ \mu \mathrm{L})$ & $14270 \pm 4570$ & $10250 \pm 4358$ & 0.87 \\
\hline C-Reactive Protein (mg/L) & $75[6,138]$ & $28[1,157]$ & $0.05^{\star}$ \\
\hline Procalcitonin (ng/dL) & $0.82[0.01,3.03]$ & $1.3[0.02,6.27]$ & 0.18 \\
\hline Hemoglobin $(\mathrm{g} / \mathrm{dL})$ & $9.0 \pm 1.2$ & $8.9 \pm 1.5$ & 0.86 \\
\hline Hematocrit (\%) & $27.2 \pm 4.9$ & $25.7 \pm 5.4$ & 0.92 \\
\hline Vasopressor support (mg/h) & $0[0,1.4]$ & $0[0,1.53]$ & 0.86 \\
\hline Clif-SOFA score & $12.6 \pm 1.9$ & $11.7 \pm 1.9$ & 0.98 \\
\hline
\end{tabular}

The median ICU length of stay was $12[5,35]$ days and overall survival was $57.1 \%(n=8)$. Seven patients (50\%) underwent liver transplantation while still in the ICU. Survival was $100 \%$ in the transplanted patients $(n=7)$ and $14.3 \%(n=1)$ in the non-transplanted patients.

\section{Discussion}

Our results show that the use of CVVHDF in combination with hemoadsorption was associated with a significant decrease in serum bilirubin and creatinine levels and an increase in urine output. The treatment was well tolerated and only three patients who previously required vasopressor support still needed noradrenaline at the end of RRT with a non-significant increase in dose. A significant decrease in platelet count was recorded in all patients.

Mortality in patients with AoCLF remains high and most of these patients require advanced treatment in dedicated liver ICUs [11]. Recent studies have shown that mortality ranges between $32 \%$ and $100 \%$ in grade 2 AoCLF and between $78 \%$ and $100 \%$ in grade 3 AoCLF[12]. This high mortality may be decreased by liver transplantation but finding a suitable liver graft is difficult and time-consuming especially in countries with a shortage of liver grafts. Thus, bridging such patients to liver transplantation remains the 
main therapeutic target of intensive care measures [13]. Survival after liver transplantation was 100\% among our patients, surprisingly higher than that reported by other studies [14] but was extremely low in patient in whom a suitable liver graft could not be found.

Current research has demonstrated that organ failure in patients with AoCLF is mainly due to translocation of bacteria and pathogen-associated molecular patters [15]. The activation of the immune system is responsible for the severe inflammatory response syndrome [16]. Our study demonstrated a significant decrease in C-reactive Protein, as a marker of systemic inflammation, but white blood cell count remained high. This may be due to the non-specific variations of leucocyte count and further studies are needed to assess the kinetics of different, more-specific molecules [17] that may aid in prognostic and futility assessment. The reason for the non-significant increase in Procalcitonin is not clear but it may be due to further bacterial translocation and subsequent infections and needs further investigation.

As mentioned, our results showed a significant decrease in bilirubin levels. This have been previously demonstrated in a small case series by Dhokia et al. [18]. To date, there are many different extracorporeal support systems [19] that proved efficient in the removal of hepatic toxins (e.g. bilirubin). But, as the use of RRT in combination with hemadsorption was associated in our study with both an improvement in liver functional tests and a decrease in inflammatory markers, it may prove to be a better bridging therapy in patients with AoCLF.

We also observed a significant decrease in platelet count. This has been previously observed in another study where patients with sepsis were treated with a combination of RRT and CytoSorb® [20]. In patients with AoCLF, the decrease in platelet count may be attributed to both intrinsic mechanism in liver disease [21] and to the use of RRT [22]. None of the patients had adverse effects due to thrombocytopenia, as no haemorrhagic complications were recorded, and we did not observe a significant decrease in haemoglobin levels and haematocrit. However, the decrease in platelet count is significant from a clinical point of few and these patients need to be carefully monitored for haemorrhagic complications and platelet transfusion should be available if bleeding is observed or if the patient must undergo invasive procedures.

Clif-SOFA is the most frequently used and Clif-C is the most sensitive score to predict 28-days mortality [23]. The mean Clif-SOFA score did not change significantly after hemadsorption. This is because the improvement in bilirubin levels and creatinine levels was counter-balanced by a decrease in platelet count.

Our study has several limitations. First, this is a small observational study. Due to the non-randomized nature of our study we cannot state with certainty that the observed benefits would not be seen with RRT alone and so the exact benefits of hemadsorption need to be confirmed by a larger randomized trial. First of all, the use of three consecutive sessions was arbitrarily chosen based on previously experience in patients with acute pancreatitis [24]. We do not know whether different number of RRT sessions or an earlier start, before the patients develops AoCLF, could have a different impact on patient outcome. 


\section{Conclusion}

To our knowledge, this is the first case series that investigated the potential benefits of hemadsorption with CytoSorb ${ }^{\circledR}$ in patients with AoCLF. Our results show improvement of liver functional tests and renal function. Thrombocytopenia remains the most important adverse effect associated with the use of hemoadsorption. Survival benefits were observed in patients who could be successfully bridged to liver transplantation.

\section{Abbreviations}

AoCLF

Acute-on-chronic liver failure

ICU

Intensive Care Unit

\section{Declarations}

- Ethics approval and consent to participate: The ethical approval and study registration were provided by the Ethical Committee of Fundeni Clinical Institute, Bucharest, Romania (16015/05/20/2016). All patients signed an informed consent.

- Consent for publication: Not applicable

- Availability of data and materials: The datasets used and/or analysed during the current study are available from the corresponding author on reasonable request

- Competing interests: The authors declare that they have no competing interests

- Funding: No funding sources to declare

- Authors' contributions: TD and PM - designed the study, collected the data and analysed the results. SD - designed the study and analysed the results. LG, IS and PI - included patients and collected the data. All authors read and approved the final manuscript

- Acknowledgements: Not applicable

\section{References}

1. Pamecha V, Kumar S, Bharathy KG. Liver transplantation in acute on chronic liver failure: challenges and an algorithm for patient selection and management. Hepatol Int. 2015;9(4):534-42.

2. Moreau R. The pathogenesis of ACLF: the inflammatory response and immune function. Semin Liver Dis. 2016;36:133-40.

3. Solé C, Solà E, Morales-Ruiz M, Fernàndez G, Huelin P, Graupera I, et al. Characterization of Inflammatory Response in Acute-on-Chronic Liver Failure and Relationship with Prognosis. Sci Rep. 2016;6:32341. 
4. Kribben A, Gerken G, Haag S, Herget-Rosenthal S, Treichel U, Betz C, et al. Effects of fractionated plasma separation and adsorption on survival in patients with acute-on-chronic liver failure. Gastroenterology. 2012;142(4):782-9.

5. Bañares R, Nevens F, Larsen FS, Jalan R, Albillos A, Dollinger M, et al. Extracorporeal albumin dialysis with the molecular adsorbent recirculating system in acute-on-chronic liver failure: the RELIEF trial. Hepatology. 2013;57(3):1153-62.

6. Stadlbauer V, Krisper P, Aigner R, Haditsch B, Jung A, Lackner C, et al. Effect of extracorporeal liver support by MARS and Prometheus on serum cytokines in acute-on-chronic liver failure. Crit Care. 2006;10(6):R169.

7. Friesecke S, Stecher SS, Gross S, Felix SB, Nierhaus A. Extracorporeal cytokine elimination as rescue therapy in refractory septic shock: a prospective single-center study. J Artif Organs. 2017;20(3):2529.

8. Träger K, Fritzler D, Fischer G, Schröder J, Skrabal C, Liebold A, et al. Treatment of PostCardiopulmonary Bypass SIRS by Hemoadsorbtion: A Case Series. Int J Artif Organs. 2016;39(3):141-6.

9. Moreau R, Jalan R, Gines P, Pavesi M, Angeli P, Cordoba J, Durand F, et al. Acute-on-chronic liver failure is a distinct syndrome that develops in patients with acute decompensation of cirrhosis. Gastroenterology. 2013;144(7):1426-37.

10. Bone RC, Balk RA, Cerra FB, Dellinger RP, Fein AM, Knaus WA, Schein RM, Sibbald WJ. Definitions for sepsis and organ failure and guidelines for the use of innovative therapies in sepsis. Chest. 1992;101(6):1644-55.

11. Vogel AS, Nahas J, Im GY. Acute-on-chronic liver failure is the most common reason for inpatient transfer to a liver transplant center and is associated with high mortality. Gastroenterology. 2017;152(5):1151.

12. Arroyo V, Moreau R. Diagnosis and prognosis of acute on chronic liver failure (ACLF) in cirrhosis. J Hepatol. 2017;66(2):451-3.

13. Choudhary NS, Saraf N, Saigal S, Soin AS. Liver Transplantation for Acute on Chronic Liver Failure. J Clin Exp Hepatol. 2017;7(3):247-52.

14. Marciano S, Mauro E, Giunta D, Torres MC, Diaz JM, Bermudez C, Gutierrez-Acevedo MN, et al. Impact of acute-on-chronic liver failure on post-transplant survival and on kidney outcomes. Eur $\mathrm{J}$ Gastroenterol Hepatol. 2019;31(9):1157-64.

15. Arroyo V, Moreau R, Kamath PS, Jalan R, Gines P, et al. Acute-on-chronic liver failure in cirrhosis. Nat Rev Dis Primers. 2016;2:16041.

16. Claria J, Stauber RE, Coenraad MJ, Moreau R, Jalan R, Pavesi M, et al. Systemic inflammation in decompensated cirrhosis: characterization and role in acute-on-chronic liver failure. Hepatology. 2016;64(4):1249-64.

17. Dirchwolf M, Podhorzer A, Marino M, Shulman C, Cartier M, Zunino M, et al. Immune dysfunction in cirrhosis: distinct cytokines phenotypes according to cirrhosis severity. Cytokine. 2016;77:14-25. 
18. Dhokia VD, Madhavan D, Austin A, Morris CG. Novel use of CytoSorb haemoadsorption to provide biochemical control in liver impairment. J Int Care Soc. 2019;20(2):174-81.

19. Wiesmann T, Hoenl D, Wulf H, Irqsusi M. Extracorporeal liver support: trending epidemiology and mortality - a nationwide database analysis 2007-2015. BMC Gastroenterol. 2019;19(1):160.

20. Kogelmann K, Jarczak D, Scheller M, Druner M. Hemoadsorbtion by CytoSorb in septic patients: a case series. Crit Care. 2017;21(1):74.

21. Fisher C, Patel VC, Stoy SH, Singanayagam A, Adelmeijer J, Wendon J, et al. Balanced haemostasis with both hypo- and hyper- coagulable features in critically ill patients with acute-on-chronic-liver failure. J Crit Care. 2018;43:54-60.

22. Ferreira JA, Johnson DW. The incidence of thrombocytopenia associated with continuous renal replacement therapy in critically ill patients. Ren Fail. 2015;37(7):1232-6.

23. Hernaez R, Sola E, Moreau R, Gines P. Acute-on-chronic liver failure: un update. Gut. 2017;66(3):54153.

24. Tomescu D, Popescu M, David C, Dima S. Clinical effects of hemoadsorption with CytoSorb® in patients with severe acute pancreatitis: A case series. Int J Artif Organs. 2019;42(4):190-3. 